\title{
The association between macronutrient intake and the metabolic syndrome and its components in type 1 diabetes
}

\author{
Aila J. Ahola ${ }^{1,2,3}$, Valma Harjutsalo ${ }^{1,2,3}$, Lena M. Thorn ${ }^{1,2,3}$, Riitta Freese ${ }^{4}$, Carol Forsblom ${ }^{1,2,3}$, \\ Sari Mäkimattila ${ }^{5}$ and Per-Henrik Groop ${ }^{1,2,3,6 *}$ on behalf of the FinnDiane Study Group \\ ${ }^{1}$ Folkhälsan Research Center, Folkhälsan Institute of Genetics, 00290 Helsinki, Finland \\ ${ }^{2}$ Abdominal Center Nephrology, University of Helsinki and Helsinki University Central Hospital, o0029 Helsinki, Finland \\ ${ }^{3}$ Research Programs Unit, Diabetes and Obesity, O0014 University of Helsinki, Finland \\ ${ }^{4}$ Department of Food and Environmental Sciences, Division of Nutrition, O0014 University of Helsinki, Finland \\ ${ }^{5}$ Abdominal Center Endocrinology, University of Helsinki and Helsinki University Central Hospital, O0029 Helsinki, Finland \\ ${ }^{6}$ Baker IDI Heart and Diabetes Institute, Melbourne, VIC 3004, Australia
}

(Submitted 1 November 2016 - Final revision received 4 January 2017 - Accepted 15 January 2017- First published online 20 February 2017)

\section{Abstract}

Diet is a major modifiable lifestyle factor that may affect the components of the metabolic syndrome. We aimed to investigate the association between relative proportions of macronutrients and the components of the metabolic syndrome in a population of individuals with type 1 diabetes. In all, 791 individuals without nephropathy, with plausible energy intake and known metabolic syndrome status, taking part in the Finnish Diabetic Nephropathy Study were included in the analyses. Dietary data were collected with a diet record. The association between the relative macronutrient intake and the outcome variables were analysed using multivariable nutrient density substitution models. The relative proportions of dietary macronutrients or fatty acids were not associated with the presence of the metabolic syndrome. In men, however, favouring carbohydrates over fats was associated with lower odds of the waist component, whereas favouring either carbohydrates or fats over proteins was associated with lower odds of the blood pressure component of the metabolic syndrome. In women, substituting carbohydrates for fats was associated with lower HDL-cholesterol concentration. Substituting carbohydrates or fats for alcohol or protein was, in men, associated with lower systolic blood pressure. To conclude, the relative distribution of macronutrients may have some relevance for the metabolic syndrome.

Key words: Type 1 diabetes: Macronutrient intakes: Metabolic syndrome: Substitution models

The metabolic syndrome is a cluster of coexisting CVD risk factors that include obesity, dyslipidaemia, hypertension and impaired glucose metabolism ${ }^{(1)}$. While the metabolic syndrome has traditionally been associated with an increased risk of type 2 diabetes $^{(2)}$, clustering of these components is also frequent among individuals with type 1 diabetes $^{(3)}$. Importantly, beyond traditional risk factors, the presence of the metabolic syndrome substantially increases the risk of cardiovascular events, and cardiovascularand diabetes-related mortality in this population ${ }^{(4)}$.

Lifestyle changes, such as dietary modifications, should be the first line of treatment upon the emergence of cardiometabolic risk factors ${ }^{(5)}$. Importantly, it has been shown that a comprehensive lifestyle intervention may improve all components of the metabolic syndrome and also prevent the occurrence of type 2 diabetes ${ }^{(6)}$. Diet is a major modifiable lifestyle factor. According to the American Diabetes Association current evidence does not, however, support one ideal dietary macronutrient distribution that would apply for all individuals with diabetes ${ }^{(7)}$. Instead, the macronutrient distribution should be based on individualised assessment of current eating patterns, preferences and metabolic goals. In practice, however, the role of carbohydrates over other macronutrients is often emphasised, as seen in the current Finnish dietary recommendations, where the recommended proportion of carbohydrates is between 45 and $60 \%$ of total energy intake ${ }^{(8)}$. For individuals with diabetes, it is important to note that the intake of carbohydrates raises the blood glucose concentrations. Moreover, the intake of carbohydrates, rather than fats, has been associated with a less favourable blood lipid panel both in a cross-sectional setting $^{(9)}$ and following a 3 -week intervention ${ }^{(10)}$.

The association between macronutrient intake and the metabolic syndrome has been evaluated in a number of studies ${ }^{(11-14)}$. No studies, to our knowledge, have however been conducted among individuals with type 1 diabetes, a population with a high

Abbreviations: E\%, percentages of total energy; LTPA, leisure-time physical activities.

* Corresponding author: P.-H. Groop, fax +358 9191 25452, email per-henrik.groop@helsinki.fi 
risk of cardiovascular complications. Moreover, the methods used in these studies differ substantially from one study to another. Importantly, when studying the associations between health markers and macronutrient intake, it should be noted that an increase in the intake of one macronutrient is, in isoenergetic conditions, always accompanied by a decrease in the intake of another macronutrient. To our knowledge, only one study has taken such an isoenergetic substitution into consideration ${ }^{(11)}$. Therefore we studied the association between macronutrient intake and the metabolic syndrome, using a multivariable nutrient density substitution model, in a population of well-defined individuals with type 1 diabetes, who are at an increased risk of CVD.

\section{Methods}

The study subjects were participants in the Finnish Diabetic Nephropathy (FinnDiane) Study. The FinnDiane Study, launched in 1997, was established with the aim to identify factors associated with the emergence of diabetic complications, especially that of diabetic nephropathy, in individuals with type 1 diabetes. Since the beginning of the study, baseline data have been collected from more than 5000 individuals. The diet sub-study was not, however, introduced until 2007. In the current cross-sectional analyses, we included all FinnDiane Study participants without diabetic nephropathy, and who had completed a minimum of 3-d diet record with plausible energy intake ((5439-14644 kJ/d) $1300-3500 \mathrm{kcal} / \mathrm{d})$, within 2 years from their study visit and whose metabolic syndrome status could be defined ( $n$ 791). The study protocol was approved by the Ethics Committee of the Helsinki University Central Hospital. Study subjects provided written informed consent before participation.

Subjects' dietary intake was evaluated using two separate methods, as previously described ${ }^{(15)}$. First, during the study visit, participants were given a self-administered diet questionnaire. With this form, we aimed at collecting data on the participants' habitual dietary intake. The form included questions regarding the consumption of some of the most typical food items in Finland such as coffee, milk, bread, fish, meat, poultry, eggs, vegetables, and fruits and berries. Next, the participants were sent a 3-d exercise and diet record together with detailed instructions. In this record participants reported all foods and drinks consumed during the allocated consecutive days. Moreover, in the same form, leisure-time physical activities (LTPA), blood glucose measurements, and insulin administrations were reported. Another 3-d exercise and food record was completed after 10 weeks from the first record. Up to two reminders were sent to the non-responders. Dietary composition was analysed using the Diet 3.2 software (version 1.4.6.2; AIVO) and, from August 2014 onwards, AivoDiet software (version 2.0.2.3; AIVO), which both are based on the Finnish National Food Composition Database, Fineli. For the current analyses, percentages of total energy (E\%) derived from the macronutrients were calculated. The proportion of energy derived from trans-fatty acids was estimated as the difference between total fat and the sum of SFA, MUFA and PUFA. Therefore, trans-fatty acids were only used to adjust the models, and not as an independent variable.
Height and weight were measured in light clothing, and these measurements were used to calculate BMI $\left(\mathrm{kg} / \mathrm{m}^{2}\right)$. Waist circumference was measured midway between the lowest rib and the iliac crest. Following a minimum of 10-min rest, blood pressure was measured while seated. The measurement was repeated after a 2-min interval, and the mean of these two measurements was used in the analyses. $\mathrm{HbA}_{1 \mathrm{c}}$ was determined locally using standardised assays. Serum lipid and lipoprotein concentrations were measured centrally at the research laboratory of the Helsinki University Central Hospital. Serum TAG concentration was measured using a Konelab $60 \mathrm{i}$ analyser (Thermo Fisher Scientific Inc.), and serum HDL-cholesterol concentration was measured using a HTS 7000 plus Bio Assay Reader (PerkinElmer Inc.). Renal status was assessed on the basis of urinary albumin excretion rate (AER) in at least two out of three timed 24 -h or overnight urine collections. Included in the analyses were individuals with normal AER $(<20 \mu \mathrm{g} / \mathrm{min}$ or $<30 \mathrm{mg} / 24 \mathrm{~h}$ ) or microalbuminuria (AER $\geq 20$ and $<200 \mu \mathrm{g} /$ $\min$ or $\geq 30$ and $<300 \mathrm{mg} / 24 \mathrm{~h}$ ). Those with diabetic nephropathy (AER $\geq 200 \mu \mathrm{g} / \mathrm{min}$ or $\geq 300 \mathrm{mg} / 24 \mathrm{~h}$, undergoing dialysis or having a kidney transplant) were excluded from the analyses, as such a diagnosis could affect both the dietary intake and the components of the metabolic syndrome. Smoking and social class (grouped as unskilled/skilled blue collar, upper/ lower white collar, farmers and others) were self-reported. Unskilled blue-collar workers were classified as low socioeconomic status.

LTPA was estimated using a validated questionnaire, as previously described ${ }^{(16)}$. As part of this questionnaire, using an eight-level scale, participants were asked to report how frequently they typically take part in LTPA (never, occasionally, 1-3 times a month, once a week, twice a week, three times a week, 4-5 times a week, more frequently). For the analyses, the population was divided into two, using the median reported frequency (those taking part in LTPA twice a week or less often, and those taking part in LTPA at least three times per week).

The criteria established by the International Diabetes Federation Task Force on Epidemiology and Prevention; National Heart, Lung, and Blood Institute; American Heart Association; World Heart Federation; International Atherosclerosis Society; and International Association for the Study of Obesity ${ }^{(1)}$ were used to define the metabolic syndrome. According to these criteria, the metabolic syndrome is present if at least three of the following criteria co-exist: waist circumference $\geq 94 \mathrm{~cm}$ in men and $\geq 80 \mathrm{~cm}$ in women; blood pressure $\geq 130 / 85 \mathrm{mmHg}$ or the use of antihypertensive medication; TAG concentration $\geq 1.70 \mathrm{mmol} / 1$ or medication to treat high TAG concentration; HDL-cholesterol concentration in men $<1.0 \mathrm{mmol} / 1$ and in women $<1.3 \mathrm{mmol} / 1$ or medication to treat reduced $\mathrm{HDL}$ cholesterol concentration; and fasting glucose $\geq 6 \cdot 11 \mathrm{mmol} / \mathrm{l}$. As all participants were diagnosed with type 1 diabetes, they were all defined to fulfil the criterion for hyperglycaemia. The metabolic syndrome and the individual criteria were treated as dichotomous variables in the analyses. Moreover, the blood pressure measurements, TAG concentration, HDL-cholesterol concentration and waist circumference were treated as continuous variables. 


\section{Statistical analyses}

Descriptive statistics are reported as mean values and standard deviations for continuous normally distributed data, median and interquartile ranges for continuous non-normally distributed data and percentages for categorical data. The respective between-group comparisons were conducted with independent samples $t$ test, Mann-Whitney $U$ test, and $\chi^{2}$ test.

To examine the associations between the macronutrient intakes and the outcome variables, multivariable nutrient density substitution models were used as described elsewhere ${ }^{(11)}$. In brief, dichotomised outcome variables were entered as dependent variables in the logistic regression analyses, whereas continuous variables were analysed using generalised linear regression. Included, in each model at a time, were all but one macronutrient (per $5 \mathrm{E} \%$ ), total energy intake, and selected variables as cofactors. The results can be interpreted as the increase or decrease in the outcome variable related to the isoenergetic ( $5 \mathrm{E} \%$ ) substitution of a given macronutrient in the model with the macronutrient left out from the model. Thus, for example, in an equation: HDL-cholesterol concentration $=\beta_{0}+\beta_{1}$ ( $5 \mathrm{E} \%$ from carbohydrates $)+\beta_{2}(5 \mathrm{E} \%$ from fats $)+\beta_{3}(5 \mathrm{E} \%$ from alcohol $)+\beta_{4}(\mathrm{~kJ}(\mathrm{kcal})), \beta_{1}$ would be interpreted as the change in the HDL-cholesterol concentration when dietary carbohydrate intake is increased by $5 \mathrm{E} \%$ at the expense of proteins.

A two-tailed $P$ value $<0.05$ was considered statistically significant. All data were analysed using IBM SPSS Statistics for Windows, version 22.0 (IBM Corp.).

\section{Results}

Those with the metabolic syndrome were older, had a longer diabetes duration, and worse glycaemic control (Table 1). Women with the metabolic syndrome were more frequently current smokers and were less physically active compared with women without the metabolic syndrome. Those with the metabolic syndrome reported lower energy intake, but the macronutrient distribution did not differ between those with and without the metabolic syndrome (Table 2).

Using the macronutrient substitution approach, the relative proportions of dietary macronutrients were not associated with the presence of the metabolic syndrome in either men or women (Table 3). However increased carbohydrate intake, at the expense of fats, was associated with lower odds of fulfilling the waist component of the metabolic syndrome, in men (as a note, the results of the multivariable models may also be interpreted reversely, thus here, increased intake of fat at the expense of carbohydrates, is associated with higher odds of fulfilling the waist component). Also in men, substituting carbohydrates or fats for proteins reduced the odds of the blood pressure component of the metabolic syndrome. In women, no associations between macronutrient substitutions and the metabolic syndrome or its components were observed. Moreover, substituting between different fatty acids was not associated with the presence of these outcome variables (online Supplementary Table S1).

In men, replacement of fat with carbohydrate was associated with lower waist circumference and lower TAG

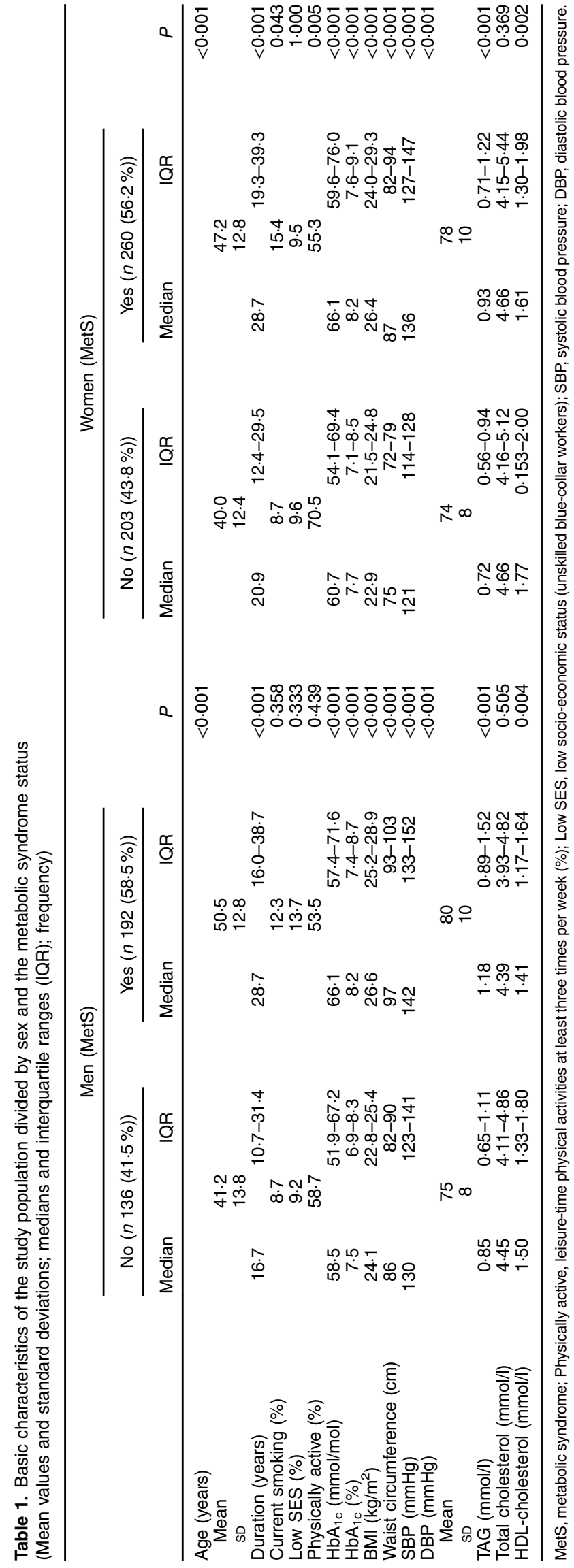




\section{Nesitish Journal of Nutrition}

Table 2. Energy and macronutrient intake of the study population divided by sex and the metabolic syndrome status

(Mean values and standard deviations; medians and interquartile ranges (IQR)

\begin{tabular}{|c|c|c|c|c|c|c|c|c|c|c|}
\hline & \multicolumn{4}{|c|}{ Men (MetS) } & \multirow[b]{3}{*}{$P$} & \multicolumn{4}{|c|}{ Women (MetS) } & \multirow[b]{3}{*}{$P$} \\
\hline & \multicolumn{2}{|c|}{ No $(n 136(41.5 \%))$} & \multicolumn{2}{|c|}{ Yes $(n 192(58.5 \%))$} & & \multicolumn{2}{|c|}{ No $(n 203(43.8 \%))$} & \multicolumn{2}{|c|}{ Yes $(n 260(56.2 \%))$} & \\
\hline & Median & IQR & Median & IQR & & Median & IQR & Median & IQR & \\
\hline $\begin{array}{l}\text { Energy (MJ) } \\
\text { Mean } \\
\text { SD }\end{array}$ & & & & & 0.011 & $7 \cdot 6$ & $6 \cdot 8-8 \cdot 6$ & $7 \cdot 2$ & $6 \cdot 4-8 \cdot 2$ & 0.003 \\
\hline $\begin{array}{l}\text { Energy (kcal) } \\
\text { Mean } \\
\text { SD }\end{array}$ & & & & & 0.011 & 1810 & $1615-2064$ & 1713 & $1519-1968$ & 0.003 \\
\hline $\begin{array}{l}\text { Carbohydrate (E\%) } \\
\text { Mean } \\
\text { SD }\end{array}$ & $44 \cdot 6$ & $40 \cdot 2-48 \cdot 7$ & $43 \cdot 3$ & $38 \cdot 9-48 \cdot 0$ & 0.097 & & & & & 0.367 \\
\hline $\begin{array}{l}\text { Protein }(\mathrm{E} \%) \\
\text { Total fat }(\mathrm{E} \%)\end{array}$ & $15 \cdot 9$ & $14 \cdot 6-18 \cdot 2$ & $16 \cdot 6$ & $14.9-18 \cdot 3$ & $\begin{array}{l}0.115 \\
0.187\end{array}$ & $16 \cdot 3$ & $14.5-18.4$ & $16 \cdot 8$ & $15 \cdot 0-18 \cdot 3$ & $\begin{array}{l}0.264 \\
0.898\end{array}$ \\
\hline $\begin{array}{l}\text { Mean } \\
\text { SD }\end{array}$ & & & & & & & & & & \\
\hline $\begin{array}{l}\text { SFA (E\%) } \\
\text { Mean } \\
\text { SD }\end{array}$ & $12 \cdot 1$ & $10 \cdot 4-14 \cdot 2$ & $12 \cdot 4$ & $10 \cdot 6-14 \cdot 4$ & 0.603 & & & & & 0.391 \\
\hline $\begin{array}{l}\text { MUFA (E\%) } \\
\text { PUFA (E\%) } \\
\text { Alcohol (E\%) }\end{array}$ & $\begin{array}{r}11.3 \\
5.4 \\
1.5\end{array}$ & $\begin{array}{c}10 \cdot 2-13 \cdot 2 \\
4 \cdot 8-6 \cdot 3 \\
0-4 \cdot 4\end{array}$ & $\begin{array}{r}11.6 \\
5.7 \\
1.4\end{array}$ & $\begin{array}{c}10 \cdot 0-13 \cdot 4 \\
4.9-6 \cdot 6 \\
0-3 \cdot 9\end{array}$ & $\begin{array}{l}0.472 \\
0.073 \\
0.861\end{array}$ & $\begin{array}{r}11.4 \\
5.8 \\
0.7\end{array}$ & $\begin{array}{c}10 \cdot 3-13 \cdot 2 \\
4 \cdot 8-6 \cdot 8 \\
0-2 \cdot 7\end{array}$ & $\begin{array}{r}11 \cdot 7 \\
5.6 \\
1.0\end{array}$ & $\begin{array}{c}10 \cdot 2-13 \cdot 4 \\
4 \cdot 9-6 \cdot 9 \\
0-3 \cdot 3\end{array}$ & $\begin{array}{l}0.733 \\
0.980 \\
0.449\end{array}$ \\
\hline
\end{tabular}

MetS, metabolic syndrome; E\%, percentage of total energy intake.

Table 3. Association between dietary macronutrient intake (substitution model) and the metabolic syndrome and its individual components (as dichotomous variables) $\dagger$ (Odds ratios and $95 \%$ confidence intervals)

\begin{tabular}{|c|c|c|c|c|c|c|c|c|c|c|c|}
\hline \multirow{2}{*}{$\begin{array}{l}\text { Macronutrients intake } \\
\text { increased (reduced) }\end{array}$} & & \multicolumn{2}{|c|}{ MetS } & \multicolumn{2}{|c|}{ Waist } & \multicolumn{2}{|c|}{ TAG } & \multicolumn{2}{|c|}{$\mathrm{HDL}$} & \multicolumn{2}{|c|}{$\mathrm{BP}$} \\
\hline & & OR & $95 \% \mathrm{Cl}$ & OR & $95 \% \mathrm{Cl}$ & OR & $95 \% \mathrm{Cl}$ & OR & $95 \% \mathrm{Cl}$ & OR & $95 \% \mathrm{Cl}$ \\
\hline \multirow[t]{2}{*}{$\mathrm{CHO}$ (protein) } & $M$ & 0.755 & $0.443,1.288$ & 0.930 & $0.558,1.551$ & 0.833 & $0.500,1.389$ & 0.706 & $0.419,1.189$ & $0.377^{*}$ & $0.160,0.888$ \\
\hline & W & 1.262 & $0.846,1.883$ & 0.990 & $0.672,1.459$ & 1.308 & $0.829,2.065$ & 1.159 & $0.763,1.761$ & 0.989 & $0.652,1.498$ \\
\hline \multirow[t]{2}{*}{$\mathrm{CHO}$ (fat) } & $M$ & 0.969 & $0.781,1.203$ & $0.795^{\star}$ & $0.640,0.988$ & 0.857 & $0.693,1.061$ & 1.003 & $0.808,1.247$ & $1 \cdot 166$ & $0.864,1.574$ \\
\hline & W & 1.135 & $0.916,1.405$ & 0.971 & $0.787,1.197$ & 1.006 & $0.799,1.266$ & 1.018 & $0.820,1.265$ & 1.242 & $0.987,1.565$ \\
\hline \multirow{2}{*}{$\mathrm{CHO}$ (alcohol) } & $M$ & 0.895 & $0.631,1.269$ & 0.951 & $0.675,1.341$ & 0.770 & $0.545,1.088$ & 0.885 & $0.622,1.260$ & 0.692 & $0.399,1.200$ \\
\hline & W & 1.014 & $0.672,1.531$ & 1.061 & $0.715,1.572$ & 0.957 & $0.632,1.450$ & 0.868 & $0.584,1.289$ & 0.928 & $0.587,1.467$ \\
\hline \multirow[t]{2}{*}{ Fat (protein) } & $M$ & 0.799 & $0.452,1.412$ & 1.178 & $0.683,2.032$ & 0.987 & $0.573,1.700$ & 0.734 & $0.422,1.276$ & $0.336^{\star}$ & $0.133,0.847$ \\
\hline & W & 1.134 & $0.745,1.727$ & 1.049 & $0.699,1.574$ & 1.318 & $0.816,2.130$ & 1.158 & $0.746,1.798$ & 0.800 & $0.513,1.247$ \\
\hline \multirow[t]{2}{*}{ Fat (alcohol) } & $\mathrm{M}$ & 0.934 & $0.642,1 \cdot 358$ & 1.199 & $0.829,1.734$ & 0.910 & $0.632,1.312$ & 0.901 & $0.618,1.314$ & 0.598 & $0.337,1.062$ \\
\hline & W & 0.929 & $0.599,1.441$ & 1.123 & $0.736,1.713$ & 0.989 & $0.627,1.560$ & 0.888 & $0.578,1.363$ & 0.754 & $0.465,1.223$ \\
\hline \multirow[t]{2}{*}{ Protein (alcohol) } & $M$ & 1.310 & $0.726,2.364$ & 1.074 & $0.616,1.873$ & 0.987 & $0.566,1.720$ & 1.468 & $0.824,2.617$ & 2.046 & $0.789,5.302$ \\
\hline & W & 0.873 & $0.494,1.543$ & 1.195 & $0.695,2.057$ & 0.795 & $0.443,1.426$ & 0.808 & $0.464,1.407$ & 0.929 & $0.499,1.729$ \\
\hline
\end{tabular}

MetS, metabolic syndrome; Waist, waist component of the metabolic syndrome; TAG, TAG component of the metabolic syndrome; HDL, HDL-cholesterol component of the metabolic syndrome; BP, blood pressure component of the metabolic syndrome; CHO, carbohydrate; M, men; W, women

* $P<0.05$.

† Logistic regression analyses. All models are adjusted for age, smoking and physical activity. In each model, a given macronutrient is included as an independent variable and one of the macronutrients (in the parentheses) is excluded from the model. The remaining macronutrients and total energy intake are included as covariates. The OR represent the increase or decrease in the risk of having the metabolic syndrome or one of its individual components when increasing the intake of the independent macronutrient by $5 \%$ of total energy, while simultaneously reducing an isoenergetic amount of the excluded macronutrient. 
concentration (Table 4). Lower HDL-cholesterol concentrations were observed when energy from alcohol was replaced with carbohydrates or fats. Lower systolic blood pressure, on the other hand, was observed when carbohydrate or fat were substituted for protein or alcohol. Finally, reducing alcohol while increasing fat intake was associated with lower diastolic blood pressure. In women, substituting carbohydrate for fats or alcohol, was associated with lower HDL-cholesterol concentration (Table 4). Substitutions between different fatty acids were not associated with any of the continuous outcome variables studied (online Supplementary Table S2).

\section{Discussion}

In the current study, in a group of well-defined individuals with type 1 diabetes, the relative distribution of dietary macronutrients was not associated with the presence of the metabolic syndrome. The substitution models showed that in men, favouring carbohydrates over fats was, however, associated with a lower prevalence of the waist component, while favouring either carbohydrates or fats over proteins was associated with a lower prevalence of the blood pressure component of the metabolic syndrome.

To our knowledge this is the first study to investigate the relative macronutrient distribution and the metabolic syndrome among individuals with type 1 diabetes. The cross-sectional nature of the study, however, prohibits any conclusions about the temporal relationships between the macronutrient intake and the health variables. Therefore, the observed results do not necessarily indicate that eating carbohydrates would be more slimming than eating fats, for example. The cause and effect could also be reversed as a low-carbohydrate-high-fat diet has successfully been used as a method of weight reduction ${ }^{(14,17)}$. Therefore, it could be possible that those with larger waist circumferences have rather switched to such a diet in order to lose weight. A meta-analysis of three population-based studies with a total of 12342 individuals, however, reported that the likelihood of being obese was the lowest in the highest quartile of total carbohydrate intake ${ }^{(18)}$. Similarly, although the current research evidence does not support it ${ }^{(19)}$, it is also possible that individuals with hypertension have reduced their protein intake, in hope of managing their blood pressure.

In isoenergetic conditions, reduced intake of a given macronutrient is accompanied by a simultaneous increase in the intake of one or several of the remaining macronutrients. As macronutrients utilise different metabolic pathways, the macronutrient composition of the diet may be of importance, particularly in studies related to the metabolic syndrome. We have been able to identify only one such previous study, in the field of the metabolic syndrome, where the macronutrient substitution was considered. Indeed, using multivariable nutrient density substitution models, in a population of individuals with high cardiovascular risk, Skilton et al. ${ }^{(11)}$ observed that an isoenergetic increase in fat intake, at the expense of protein, was associated with a decrease in the prevalence of the metabolic syndrome. Reduced risk of the metabolic syndrome was also observed when carbohydrates were substituted for either fats or proteins. 
Regarding the continuous outcome variables, in the present study, favouring carbohydrates over fats was, in women, associated with lower HDL-cholesterol concentrations. This observation was also made, not only in the study by Skilton et $a l .{ }^{(11)}$, but also in another study among healthy individuals free of any major medical problems ${ }^{(20)}$. Consistent with our observations among men, Skilton et $a l .{ }^{(11)}$ reported that substituting carbohydrates for fats was associated with lower odds of fulfilling the waist circumference criterion of the metabolic syndrome. In their study, however, favouring carbohydrates or fats over proteins was also associated with reduced odds of the waist component. Moreover, in their population, consumption of up to 1 standard alcohol drink/d was associated with a lower prevalence of the metabolic syndrome when compared with non-drinkers. Finally, similar to us, Skilton et al. observed no association between the proportion of dietary fatty acids and the metabolic syndrome or its components.

In the current study, substituting carbohydrates (in men and women) or fats (in men) for alcohol was also associated with lower HDL-cholesterol concentrations. A beneficial effect of moderate alcohol consumption on HDL-cholesterol has also previously been reported $^{(21)}$. On the other hand, higher alcohol or protein consumption, over fats or carbohydrates was, in men, associated with higher systolic blood pressure. The positive association between alcohol intake and blood pressure, has also been previously reported ${ }^{(22)}$. Finally, substitution of carbohydrates with fats was associated with lower TAG concentrations, in men. This observation is not in concordance with any previous reports, which have shown higher TAG concentrations with higher carbohydrate intakes ${ }^{(11,14)}$. While the reason behind this difference is not known, it can be speculated that different study populations might contribute. Compared with other populations, those with type 1 diabetes may, for example, be more readily treated with lipid lowering agents. Moreover, as previously shown, the mean intake of carbohydrates in the FinnDiane population is low ${ }^{(15)}$, and could be lower than in other populations.

A number of other studies, although not using the multivariable nutrient density substitution model, have also been conducted to reveal the associations between dietary macronutrient intake and the metabolic syndrome. In one such study, Freire et $a l^{(13)}$ observed that total fat intake was positively associated with the presence of the metabolic syndrome in Japanese Brazilians. In a population of American Indians, PUFA intake in women and simple carbohydrate intake in men was associated with lower and higher prevalence of the metabolic syndrome, respectively ${ }^{(23)}$. Yet in another study, a wealthier intake of PUFA and fibre, among subjects with high CVD risk, was associated with reduced odds of the metabolic syndrome ${ }^{(24)}$. Finally, in the cross-sectional analyses of the Framingham Offspring Study, total carbohydrate intake, measured with a semi quantitative FFQ, was not associated with the prevalence of the metabolic syndrome $e^{(12)}$

There are a number of limitations related to the current study that need to be acknowledged. First, the use of diet record has its limitations. The very act of reporting dietary intake may result in over- or under-reporting food items that are considered healthy and unhealthy, respectively. In addition, although truthfully reporting their dietary intake, during the allocated days, subjects may still have altered their food intake for the duration of record-keeping to reflect more socially acceptable dietary habits. Should these phenomena have taken place, they have most likely diluted the current observations. Second, as is the case in many other studies, our study is limited by potential selection bias. Thus, participants in health related studies are likely to be over-represented by health-conscious individuals, with healthier lifestyle and, in our case, potentially less prevalent metabolic syndrome. Again, should this have taken place, it would have only diluted the results. Third, typical to observational studies, we cannot rule out the possibility of residual confounding. Fourth, while the use of a substitution model is justified when studying the health effects of different macronutrients in isoenergetic conditions, it has to be acknowledged that this approach is only a mathematical model for dietary intake and not a real-life condition. Moreover, diet is more than just macronutrients. In these analyses the intake of items such as fibre, vitamins and minerals from different food sources are not taken into account. Finally, this was a crosssectional study, which does not reveal causalities, but only associations. In the future, the prospective associations between the dietary intake and the metabolic syndrome will be assessed.

In conclusion, relative macronutrient distribution was not associated with the presence of the metabolic syndrome in type 1 diabetes. In men, a diet with more abundant intake of carbohydrates over fats was associated with lower odds of fulfilling the waist component, while substituting carbohydrates or fats for proteins was associated with lower odds of fulfilling the blood pressure component of the metabolic syndrome. To reveal potential causalities between different macronutrient intakes and health variables, prospective studies in real-life circumferences are needed.

\section{Acknowledgements}

The skilled technical assistance of Anna Sandelin, Mira Korolainen, Jaana Tuomikangas and Satu Kinnunen is gratefully acknowledged. The authors also acknowledge all the physicians and nurses at each centre participating in the collection of patients data (online Supplementary Material).

This study was supported by grants from Academy of Finland, Novo Nordisk Foundation, Signe and Ane Gyllenberg Foundation, Folkhälsan Research Foundation, Wilhelm and Else Stockmann Foundation, Liv och Hälsa Foundation, the Helsinki University Central Hospital Research Funds (EVO). Funding agencies did not contribute to the study design, conduct of the study, data analysis, interpretation of findings, writing of the manuscript or in the decision to submit the manuscript for publication.

All authors took part in designing the research. A. J. A. analysed the data, and wrote the first draft of the paper. A. J. A. and P.-H. G. had the primary responsibility for the final content. All authors have contributed to the writing and approved the final manuscript.

P.-H. G. has received research grants from Eli Lilly and Roche, is an advisory board member for AbbVie, AstraZeneca, BoehringerIngelheim, Cebix, Eli Lilly, Janssen, MSD, Medscape, Novartis and 
Sanofi. He has received lecture fees from AstraZeneca, Boehringer-Ingelheim, Eli Lilly, Genzyme, MSD, Novartis, Novo Nordisk and Sanofi. All other authors declare that there are no conflicts of interest.

\section{Supplementary material}

For supplementary material referred to in this article, please visit https://doi.org/10.1017/S0007114517000198

\section{References}

1. Alberti KG, Eckel RH, Grundy SM, et al. (2009) Harmonizing the metabolic syndrome: a joint interim statement of the International Diabetes Federation Task Force on Epidemiology and Prevention; National Heart, Lung, and Blood Institute; American Heart Association; World Heart Federation; International Atherosclerosis Society; and International Association for the Study of Obesity. Circulation 120, 1640-1645.

2. Aschner P (2010) Metabolic syndrome as a risk factor for diabetes. Expert Rev Cardiovasc Ther 8, 407-412.

3. Thorn LM, Forsblom C, Fagerudd J, et al. (2005) Metabolic syndrome in type 1 diabetes: association with diabetic nephropathy and glycemic control (the FinnDiane study). Diabetes Care 28, 2019-2024.

4. Thorn LM, Forsblom C, Waden J, et al. (2009) Metabolic syndrome as a risk factor for cardiovascular disease, mortality, and progression of diabetic nephropathy in type 1 diabetes. Diabetes Care 32, 950-952.

5. Aronne LJ (2007) Therapeutic options for modifying cardiometabolic risk factors. Am J Med 120, Suppl. 1, S26-S34.

6. Tuomilehto J, Lindstrom J, Eriksson JG, et al. (2001) Prevention of type 2 diabetes mellitus by changes in lifestyle among subjects with impaired glucose tolerance. N Engl J Med $\mathbf{3 4 4}$, 1343-1350.

7. Evert $\mathrm{AB}$, Boucher JL, Cypress M, et al. (2014) Nutrition therapy recommendations for the management of adults with diabetes. Diabetes Care 37, Suppl. 1, S120-S143.

8. National Nutrition Council (2014). Finnish dietary recommendations 2014. http://www.ravitsemusneuvottelukunta.fi/ files/attachments/fi/vrn/ravitsemussuositukset_2014_fi_web.3. pdf (accessed November 2016).

9. Wiltshire EJ, Hirte C \& Couper JJ (2003) Dietary fats do not contribute to hyperlipidemia in children and adolescents with type 1 diabetes. Diabetes Care 26, 1356-1361.

10. Jeppesen J, Schaaf P, Jones C, et al. (1997) Effects of low-fat, high-carbohydrate diets on risk factors for ischemic heart disease in postmenopausal women. Am J Clin Nutr 65 $1027-1033$.

11. Skilton MR, Laville M, Cust AE, et al. (2008) The association between dietary macronutrient intake and the prevalence of the metabolic syndrome. Br J Nutr 100, 400-407.

12. McKeown NM, Meigs JB, Liu S, et al. (2004) Carbohydrate nutrition, insulin resistance, and the prevalence of the metabolic syndrome in the Framingham Offspring Cohort. Diabetes Care 27, 538-546.

13. Freire RD, Cardoso MA, Gimeno SG, et al. (2005) Dietary fat is associated with metabolic syndrome in Japanese Brazilians. Diabetes Care 28, 1779-1785.

14. Volek JS, Phinney SD, Forsythe CE, et al. (2009) Carbohydrate restriction has a more favorable impact on the metabolic syndrome than a low fat diet. Lipids $\mathbf{4 4}, 297-309$.

15. Ahola AJ, Mikkilä V, Mäkimattila S, et al. (2012) Energy and nutrient intakes and adherence to dietary guidelines among Finnish adults with type 1 diabetes. Ann Med 44, 73-81.

16. Wadén J, Tikkanen H, Forsblom C, et al. (2005) Leisure time physical activity is associated with poor glycemic control in type 1 diabetic women: the FinnDiane study. Diabetes Care 28, $777-782$.

17. Gardner CD, Kiazand A, Alhassan S, et al. (2007) Comparison of the Atkins, Zone, Ornish, and LEARN diets for change in weight and related risk factors among overweight premenopausal women: the A TO Z Weight Loss Study: a randomized trial. JAMA 297, 969-977.

18. Kaartinen NE, Knekt P, Kanerva N, et al. (2016) Dietary carbohydrate quantity and quality in relation to obesity: a pooled analysis of three Finnish population-based studies. Scand J Public Health 44, 385-393.

19. Santesso N, Akl EA, Bianchi M, et al. (2012) Effects of higherversus lower-protein diets on health outcomes: a systematic review and meta-analysis. Eur J Clin Nutr 66, 780-788.

20. Abbasi F, McLaughlin T, Lamendola C, et al. (2000) High carbohydrate diets, triglyceride-rich lipoproteins, and coronary heart disease risk. Am J Cardiol 85, 45-48.

21. Brinton EA (2012) Effects of ethanol intake on lipoproteins. Curr Atheroscler Rep 14, 108-114.

22. Savica V, Bellinghieri G \& Kopple JD (2010) The effect of nutrition on blood pressure. Annu Rev Nutr 21, 365-401.

23. Eilat-Adar S, Xu J, Goldbourt U, et al. (2008) Sex may modify the effects of macronutrient intake on metabolic syndrome and insulin resistance in American Indians: the strong heart study. J Am Diet Assoc 108, 794-802.

24. Cabello-Saavedra E, Bes-Rastrollo M, Martinez JA, et al. (2010) Macronutrient intake and metabolic syndrome in subjects at high cardiovascular risk. Ann Nutr Metab 56, 152-159. 